\title{
Recent Advances of Therapeutic Targets for the Treatment of Periodontal Disease
}

\author{
Woo Jin Kim ${ }^{1}$, Yunjo Soh ${ }^{2, *}$ and Seok-Mo Heo ${ }^{3,4, *}$ \\ ${ }^{1}$ Department of Molecular Genetics, School of Dentistry and Dental Research Institute, Seoul National University, Seoul 03080 , \\ ${ }^{2}$ Laboratory of Pharmacology, School of Pharmacy and Institute of New Drug Development, Jeonbuk National University, Jeonju 54896, \\ ${ }^{3}$ Department of Periodontology, School of Dentistry, Jeonbuk National University, Jeonju 54896, \\ ${ }^{4}$ Research Institute of Clinical Medicine of Jeonbuk National University, Jeonju 54907, Republic of Korea
}

\begin{abstract}
Periodontal disease is primarily associated with bacterial infection such as dental plaque. Dental plaque, an oral biofilm harboring a complex microbial community, can cause various inflammatory reactions in periodontal tissue. In many cases, the local bacterial invasion and host-mediated immune responses lead to severe alveolar bone destruction. To date, plaque control, non-surgical, and surgical interventions have been the conventional periodontal treatment modalities. Although adjuvant therapies including antibiotics or supplements have accompanied these procedures, their usage has been limited by antibiotic resistance, as well as their partial effectiveness. Therefore, new strategies are needed to control local inflammation in the periodontium and host immune responses. In recent years, target molecules that modulate microbial signaling mechanisms, host inflammatory substances, and bone immune responses have received considerable attention by researchers. In this review, we introduce three approaches that suggest a way forward for the development of new treatments for periodontal disease; (1) quorum quenching using quorum sensing inhibitors, (2) inflammasome targeting, and (3) use of FDA-approved anabolic agents, including Teriparatide and sclerostin antibody.
\end{abstract}

Key Words: Quorum sensing and quenching, Inflammasome, Teriparatide, Sclerostin antibody, Periodontitis, Parathyroid hormone

\section{INTRODUCTION}

Periodontal disease is caused primarily by oral bacterial infections (Loos and Van Dyke, 2020). Dental plaque, a biofilm comprised of a complex microbial community which grows on tooth surfaces, may trigger an inflammatory response in periodontal tissue (Filoche et al., 2010). Periodontal inflammation may be accompanied by bleeding, swelling, or pain. Gingivitis is a common reversible inflammation of the soft tissues of the gingiva that affects up to $95 \%$ of the world's population (Albandar and Rams, 2002). Periodontitis is caused by bacterial invasion or bacterial toxins, but the pathology of the disease is mainly affected by host immune reaction (Filoche et al., 2010; Loos and Van Dyke, 2020). The response of a particular host to these bacteria depends on genetic, immunological and environmental factors (Loos and Van Dyke, 2020). While gingivitis can be resolved with meticulous personal oral hygiene ac- companied by plaque removal, periodontitis is an irreversible disease that affects $50 \%$ of the world's population (Albandar and Rams, 2002). Moreover, prolonged and untreated gingival inflammation may eventually lead to alveolar bone destruction and tooth loss (Caton et al., 2018). For that reason, prevention, early diagnosis, and timely treatment are recommended as a matter of course (Albandar and Rams, 2002). The current diagnostic classification of periodontitis is divided into four stages and of three grades according to severity and progression respectively (Caton et al., 2018). Once a patient's case is classified, an appropriate periodontal treatment modality can be applied. To date, conventional periodontal treatments include non-surgical and surgical interventions (Deas et al., 2016) paired with adjunctive pharmaceuticals (Sheridan et al., 2015) or nutraceuticals (Laleman and Teughels, 2020). In this review, we bring forth an overview of recent advances in the identification of therapeutic targets for the development

\section{Open Access https://doi.org/10.4062/biomolther.2021.001}

This is an Open Access article distributed under the terms of the Creative Commons Attribution Non-Commercial License (http://creativecommons.org/licenses/by-nc/4.0/) which permits unrestricted non-commercial use, distribution, and reproduction in any medium, provided the original work is properly cited.
Received Jan 4, 2021 Revised Jan 25, 2021 Accepted Jan 29, 2021 Published Online Mar 17, 2021

*Corresponding Authors

E-mail: neoheo@jbnu.ac.kr (Heo SM), ysoh@jbnu.ac.kr (Soh Y) Tel: +82-63-250-2018 (Heo SM), +82-63-270-4038 (Soh Y) Fax: +82-63-250-2259 (Heo SM), +82-63-270-4038 (Soh Y) 

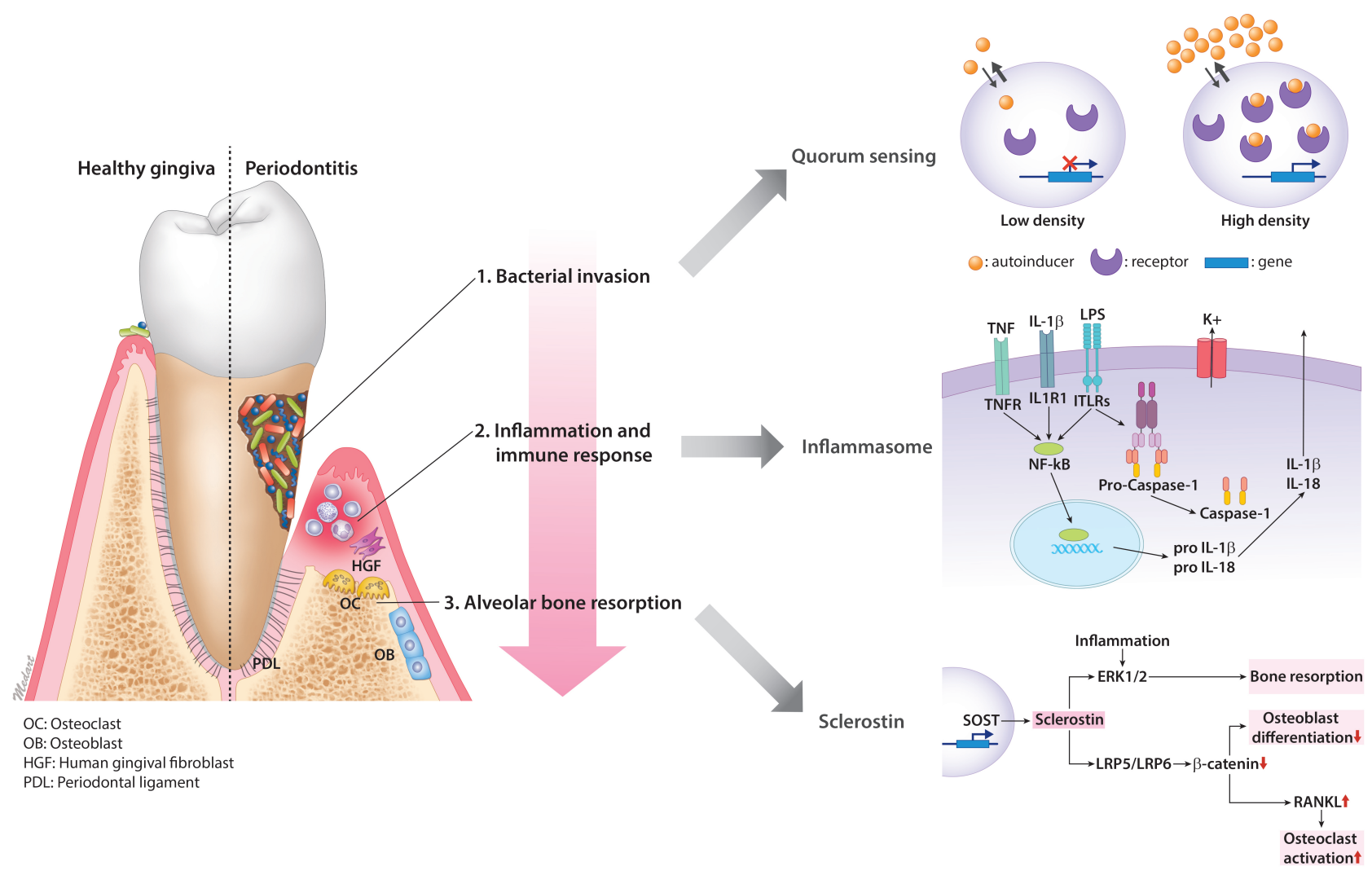

Fig. 1. Overview of periodontal disease and therapeutic targets.

Table 1. Pharmaceutical targets for periodontal disease

\begin{tabular}{|c|c|c|}
\hline Classification & Target & Mechanism \\
\hline $\begin{array}{l}\text { Quorum } \\
\text { sensing }\end{array}$ & $\begin{array}{l}\text { LuxI/Lux R type system } \\
\text { Oligopeptide/two component type system } \\
\text { Lux S encoded autoinducer- } 2\end{array}$ & $\begin{array}{l}\text { Usage of acyl homoserine lactones as an autoinducer in } G(-) \text { bacteria } \\
\text { Usage of amino acids and short peptide derivatives as an autoinducer in } G(+) \text { bacteria } \\
\text { Act on both } G(+) \text { and } G(-) \text { bacteria }\end{array}$ \\
\hline $\begin{array}{l}\text { Quorum } \\
\text { quenching }\end{array}$ & $\begin{array}{l}\text { Acylase, Lactonase, Oxyreductase } \\
\text { S-adenosyl methionine } \\
\text { S-adenosyl cysteine }\end{array}$ & $\begin{array}{l}\text { Inactivation of } \mathrm{AHL} \text { in } \mathrm{G}(-) \text { bacterial by enzymatic degradation of signaling molecules } \\
\text { Inhibition of signal generation by blockage of } \mathrm{AHL} \text { synthesis }\end{array}$ \\
\hline $\begin{array}{l}\text { Inflam- } \\
\text { masome }\end{array}$ & $\begin{array}{l}\text { Sulforaphane } \\
\text { Glyburide } \\
\text { NSAIDs of fenamate class }\end{array}$ & $\begin{array}{l}\text { Inhibits caspase- } 1 \text { proteolytic activation, IL-1 } \beta \text { maturation and secretion down- } \\
\text { stream of several inflammasomes } \\
\text { Inhibit NLRP3 inflammasome activation and IL-1 } \beta \text { secretion } \\
\text { Inhibit NLRP3 inflammasome activation }\end{array}$ \\
\hline Sclerostin & $\begin{array}{l}\text { Sclerostin antibody } \\
\text { SOST gene }\end{array}$ & $\begin{array}{l}\text { Neutralizing antibody to inhibit sclerostin action, ex) Romosozumab } \\
\text { Sclerostin in encoded by SOST gene }\end{array}$ \\
\hline
\end{tabular}

$\mathrm{AHL}$, acyl homoserine lactone.

of novel treatment modalities in periodontal disease (Fig. 1). Table 1 summarizes the proposed pharmaceutical targets and mechanism of actions for periodontal disease.

\section{Bacterial invasion: quorum sensing and quenching}

Resistance to antibiotics is the major side effect in treating periodontal disease. Excessive use of antibiotics can selectively increase several antibiotic-resistant periodontal microorganisms (Sheridan et al., 2015). Thus, to remove periodontal pathogens, antibiotics have been used limitedly as an adjunct therapy combined with mechanical interventions including scaling and root planing (Deas et al., 2016). Fortunately, new targets for inhibiting bacterial pathogenicity have been discovered. Microorganisms monitor and modulate their population density by secreting chemical signaling molecules, called autoinducers (Federle and Bassler, 2003). This phenomenon is known as 'quorum sensing (QS)' that is defined as the ability responding to fluctuations in population density with altera- 
tions in the regulation of gene expression. Because intra- and inter-bacterial communication is key to biofilm formation, terminating this communication is one means of controlling biofilm formation and virulence factor expression. The act of inhibiting QS by enzymatic hydrolysis of autoinducer is referred to as 'quorum quenching'. Gram-negative bacteria use N-acylhomoserine lactone (AHL) while gram-positive bacteria use peptides as an autoinducer. Therefore, quorum sensing inhibitors (QSIs), which are mostly produced naturally by plants, algae, fungi, etc., are considered suitable candidates to achieve this quenching effect (Venkatramanan et al., 2020). Researchers are optimistic that QSIs, although still in the research stage, will open the door to new drugs for treatment of chronic periodontal disease. There is certainly cause for optimism, as a few pre-clinical studies have already provided some evidence that QSIs are effective at inhibiting plaque biofilm formation and controlling periodontal disease (Biradar and Devi, 2011; Yada et al., 2015).

\section{Inflammation \& immune response: inflammasome}

Injury of the human body results in a sequential cascade of events that includes immune and inflammatory responses. Inflammasome, a cytosolic multiprotein oligomer of the innate immune system, mediates the body's inflammatory response to injury. Normal inflammasome assembly induces proteolytic cleavage, maturation, and production of pro-inflammatory cytokine such as interleukin, while abnormal inflammasome activation may result in the development of various diseases, including cancer, autoimmune, and metabolic disease. During normal inflammasome production, the priming procedure is initiated by stimulation of nuclear factor (NF)-kB via lipopolysaccharide (LPS) or tumor necrosis factor-b induced expression of inflammasome's components. After priming is completed and once an activation signal is received, inflammasome multimerizes and autoactivates caspase-1. Different signals are used to activate the production of over 10 distinct types of inflammasome. Among these, NODs and NACHT-leucine-rich repeats (NLRs), absent in melanoma 2-like receptor (ALR), and Pyrin inflammasome are associated with periodontal disease pathogenesis (Marchesan et al., 2020).

NLR inflammasome (NLRP1 and NLRP3): NODs and NACHT leucine rich repeats (NLRs) are a large family of intracellular proteins regulating innate immune responses. In one clinical study, NLRP1 was found to be present at low levels in healthy gingiva and at elevated levels in the epithelium and connective tissue of aggressive periodontitis (Xue et al., 2015). In another study, the presence of NLRP3 was significantly raised in patients suffering from periodontitis (Isaza-Guzman et al., 2017). However, the role of NLR inflammasomes in periodontal disease progression is still unclear, with further study needed before these proteins can be assessed for their potential to play a role in the treatment of periodontitis.

ALR inflammasome (IFI16 and AIM2): Absent in melanoma 2-like receptors (ALRs) are a family of DNA-binding proteins containing DNA-binding domains and pyrin. In one clinical study, levels of absent in melanoma 2 (AIM2) were elevated in patients experiencing chronic periodontitis (Xue et al., 2015), and genetic variants in interferon $\gamma$-inducible protein 16 (IFI16) and AIM2 were found to correlate with the presence of periodontal disease (Marchesan et al., 2017).

\section{Bone regeneration: anabolic agents}

Teriparatide (FORTEO $\left.{ }^{\circledR}\right)$ : Teriparatide (FORTEO ${ }^{\circledR}$ ) which consists of the bioactive portion of parathyroid hormone (PTH), was the first anabolic drug approved by the FDA to treat osteoporosis. Because PTH plays a role in the WNT/ $\beta$-catenin signaling that downregulates sclerostin, which is an inhibitor of WNT-LRP5/6, and stimulates bone formation, Teriparatide is a research target among periodontists as a potential means of achieving alveolar bone regeneration (Jilka et al., 1999; Kramer et al., 2010). Teriparatide activates a signaling cascade that includes protein kinase-1, cyclic adenosine monophosphate, and protein kinase (Fujita et al., 2002). A number of preclinical studies have shown that teriparatide has potential as a therapeutic target for alveolar bone defects (Jung et al., 2007). Moreover, clinical studies involving the systematic administration of teriparatide for periodontal tissue regeneration resulted in successful outcomes of significant bone formation (Bashutski et al., 2010, 2012). In Bashutski et al. (2010)'s clinical study, a daily subcutaneous injection of teriparatide $(20 \mu \mathrm{g})$ over the course of 6 weeks resulted in significant bone formation in the osseous defect, accompanied by a $33 \%$ reduction of periodontal probing depth, and a $22 \%$ gain in clinical attachment level. In another study by Kuchler et al. (2011), shortterm (28 days) daily systemic administration of teriparatide positively affected the osseointegration of a titanium implant. While the clinical effects of teriparatide have been observed, the inconvenience of daily injections and the risk of osteosarcoma has prompted a search for new candidate drugs that would offer similar benefits without these drawbacks, including sclerostin antibodies (Suen and Qin, 2016). Alternatively, teriparatide may be incorporated into current treatment regimens for periodontal disease and dental implants if new methods of oral or local drug delivery are developed.

Sclerostin antibody: Second only to bone morphogenetic proteins, the $\mathrm{Wnt} / \beta$-catenin signaling pathway has received much attention due to its importance in osteoblast differentiation and bone formation (Baron and Kneissel, 2013). Similar to other signaling mechanisms, Wnt signaling is also regulated by an agonist and antagonist. Sclerostin, encoded by the SOST gene, is primarily expressed in the osteocyte and achieves an anti-anabolic effector by inhibiting Wnt signaling. Historically, it was believed that sclerostin was a BMP antagonist (Winkler et al., 2003), however, it was subsequently determined that sclerostin bonded to the WNT co-receptor (low density lipoprotein receptor-related protein 5/6 (LRP5/6)) and thus inhibited the Wnt signaling pathway (Li et al., 2005). Boyden et al. (2002) identified that mutations in LRP5 decreased sclerostin binding, and that the resulting lack of sclerostin induced high bone mass (a condition referred to as sclerosteosis) (MIM269500). These findings generated significant interest in both the academic and private sectors, where researchers have spent the last decade exploring the role of WNT signaling in bones through a wealth of genetic studies of mice and humans. All of these studies have confirmed the importance of this pathway in bone biology and disease. Building on these studies, sclerostin antibody ( $\mathrm{Scl}-\mathrm{Ab}$ ) has been widely studied as a bone anabolic agent for possible treatment of osteoporosis, and numerous preclinical and clinical studies have proved its effects on bone formation (Ominsky et al., 2010; Padhi et al., 2011). As alveolar bone and skeletal bone rely on a similar mechanism during bone remodeling, including the balance of osteoblast and osteoclast, drugs that target 
skeletal bone may be suitable candidates for treatment of periodontal disease. Balli et al. (2015) reported that sclerostin and the sclerostin:RANKL ratio were higher in the gingival crevicular fluid of periodontitis patients, and some pre-clinical studies have shown that sclerostin inhibition decreased bone resorption and promoted bone regeneration in animal periodontitis models (Ren et al., 2015; Yao et al., 2020). For the same reasons that sclerostin inhibition using Scl-Ab has proven helpful to patients with osteoporosis, sclerostin modulation may prove to be an effective pharmaceutical target for the treatment of periodontal disease.

\section{CONCLUSION AND FUTURE DIRECTION}

Periodontitis is a bacterial-associated and host-mediated multifactorial inflammatory disease. Although the overall phenotype of chronic periodontitis may be similar across patients, the underlying causes of the disease varies from person to person. Traditional periodontal treatment fails primarily when local inflammation caused by bacterial invasion is met with an uncontrolled host immune response. Novel responses to periodontitis will require an understanding of individual molecular pathogenesis and the development of target-oriented therapeutic drugs.

The recent focus on inhibiting plaque biofilm formation with QSIs may result in the development of new drugs for periodontal treatment. Moreover, as inflammasomes and their components (NLR, ALR, and Pyrin) are associated with the onset of periodontal disease, drugs that directly target the inflammasome and relieve periodontal inflammation may be developed. Finally, two anabolic agents that inhibit bone loss and promote bone regeneration may be useful in treating advanced cases of periodontitis. Preclinical studies have shown that Teriparatide and sclerostin antibody are both effective in periodontitis.

Of course, the safety and effectiveness of any of these drugs must be verified before being widely adopted. As this review has shown, however, there is a sound argument to be made that these potential treatments are ready for the next stage of testing; multicenter human clinical trial studies.

\section{ACKNOWLEDGMENTS}

This work was supported by Fund of Biomedical Research Institute, Jeonbuk National University Hospital (SH) and by the Technology Innovation Program (20012892) funded by the Ministry of Trade, Industry \& Energy (MOTIE) of Korea (YS).

\section{REFERENCES}

Albandar, J. M. and Rams, T. E. (2002) Global epidemiology of periodontal diseases: an overview. Periodontol. 2000 29, 7-10.

Balli, U., Aydogdu, A. , Dede, F. O., Turer, C. C. and Guven, B. (2015) Gingival crevicular fluid levels of sclerostin, osteoprotegerin, and receptor activator of nuclear factor-kappa B ligand in periodontitis. J. Periodontol. 86, 1396-1404.

Baron, R. and Kneissel, M. (2013) WNT signaling in bone homeostasis and disease: from human mutations to treatments. Nat. Med. 19, 179-192.

Bashutski, J. D., Eber, R. M., Kinney, J. S., Benavides, E., Maitra, S.,
Braun, T. M., Giannobile, W. V. and McCauley, L. K. (2010) Teriparatide and osseous regeneration in the oral cavity. N. Engl. J. Med. 363, 2396-2405.

Bashutski, J. D., Kinney, J. S., Benavides, E., Maitra, S., Braun, T. M., Giannobile, W. V., McCauley, L. K. and Eber, R. M. (2012) Systemic teriparatide administration promotes osseous regeneration of an intrabony defect: a case report. Clin. Adv. Periodontics 2, 66-71.

Biradar, B. and Devi, P. (2011) Quorum sensing in plaque biofilms: challenges and future prospects. J. Contemp. Dent. Pract. 12, 479485.

Boyden, L. M., Mao, J. H., Belsky, J., Mitzner, L., Farhi, A., Mitnick, M. A., Wu, D. Q., Insogna, K. and Lifton, R. P. (2002) High bone density due to a mutation in LDL-receptor-related protein 5. N. Engl. J. Med. 346, 1513-1521.

Caton, J. G., Armitage, G., Berglundh, T., Chapple, I. L. C., Jepsen, S., Kornman, K. S., Mealey, B. L., Papapanou, P. N., Sanz, M. and Tonetti, M. S. (2018) A new classification scheme for periodontal and peri-implant diseases and conditions - Introduction and key changes from the 1999 classification. J. Periodontol. 89, S1-S8.

Deas, D. E., Moritz, A. J., Sagun, R. S., Jr., Gruwell, S. F. and Powell, C. A. (2016) Scaling and root planing vs. conservative surgery in the treatment of chronic periodontitis. Periodontol. 2000 71, 128139.

Federle, M. J. and Bassler, B. L. (2003) Interspecies communication in bacteria. J. Clin. Invest. 112, 1291-1299.

Filoche, S., Wong, L. and Sissons, C. H. (2010) Oral biofilms: emerging concepts in microbial ecology. J. Dent. Res. 89, 8-18.

Fujita, T., Meguro, T., Fukuyama, R., Nakamuta, H. and Koida, M. (2002) New signaling pathway for parathyroid hormone and cyclic AMP action on extracellular-regulated kinase and cell proliferation in bone cells. Checkpoint of modulation by cyclic AMP. J. Biol. Chem. 277, 22191-22200.

Isaza-Guzman, D. M., Medina-Piedrahita, V. M., Gutierrez-Henao, C. and Tobon-Arroyave, S. I. (2017) Salivary levels of NLRP3 inflammasome-related proteins as potential biomarkers of periodontal clinical status. J. Periodontol. 88, 1329-1338.

Jilka, R. L., Weinstein, R. S., Bellido, T., Roberson, P., Parfitt, A. M. and Manolagas, S. C. (1999) Increased bone formation by prevention of osteoblast apoptosis with parathyroid hormone. J. Clin. Invest. 104, 439-446.

Jung, R. E., Cochran, D. L., Domken, O., Seibl, R., Jones, A. A., Buser, D. and Hammerle, C. H. (2007) The effect of matrix bound parathyroid hormone on bone regeneration. Clin. Oral Implants Res. 18, 319-325.

Kramer, I., Keller, H., Leupin, O. and Kneissel, M. (2010) Does osteocytic SOST suppression mediate PTH bone anabolism? Trends Endocrinol. Metab. 21, 237-244.

Kuchler, U., Luvizuto, E. R., Tangl, S., Watzek, G. and Gruber, R. (2011) Short-term teriparatide delivery and osseointegration: a clinical feasibility study. J. Dent. Res. 90, 1001-1006.

Laleman, I. and Teughels, W. (2020) Novel natural product-based oral topical rinses and toothpastes to prevent periodontal diseases. Periodontol. 2000 84, 102-123.

Li, X. F., Zhang, Y. Z., Kang, H. S., Liu, W. Z., Liu, P., Zhang, J. G., Harris, S. E. and Wu, D. Q. (2005) Sclerostin binds to LRP5/6 and antagonizes canonical Wnt signaling. J. Biol. Chem. 280, 1988319887.

Loos, B. G. and Van Dyke, T. E. (2020) The role of inflammation and genetics in periodontal disease. Periodontol. 2000 83, 26-39.

Marchesan, J. T., Girnary, M. S., Moss, K., Monaghan, E. T., Egnatz, G. J., Jiao, Y., Zhang, S., Beck, J. and Swanson, K. V. (2020) Role of inflammasomes in the pathogenesis of periodontal disease and therapeutics. Periodontol. 2000 82, 93-114.

Marchesan, J. T., Jiao, Y., Moss, K., Divaris, K., Seaman, W., WebsterCyriaque, J., Zhang, S., Yu, N., Song, C., Bencharit, S., Teles, R. and Offenbacher, S. (2017) Common polymorphisms in IFI16 and AIM2 genes are associated with periodontal disease. J. Periodontol. 88, 663-672.

Ominsky, M. S., Vlasseros, F., Jolette, J., Smith, S. Y., Stouch, B., Doellgast, G., Gong, J. H., Gao, Y. M., Cao, J., Graham, K., Tipton, B., Cai, J., Deshpande, R., Zhou, L., Hale, M. D., Lightwood, D. J., Henry, A. J., Popplewell, A. G., Moore, A. R., Robinson, M. K., 
Lacey, D. L., Simonet, W. S. and Paszty, C. (2010) Two doses of sclerostin antibody in cynomolgus monkeys increases bone formation, bone mineral density, and bone strength. J. Bone Miner. Res. 25, 948-959.

Padhi, D., Jang, G., Stouch, B., Fang, L. A. and Posvar, E. (2011) Single-dose, placebo-controlled, randomized study of AMG 785, a sclerostin monoclonal antibody. J. Bone Miner. Res. 26, 19-26.

Ren, Y. S., Han, X. L., Ho, S. P., Harris, S. E., Cao, Z. G., Economides, A. N., Qin, C. L., Ke, H. Z., Liu, M. and Feng, J. Q. (2015) Removal of SOST or blocking its product sclerostin rescues defects in the periodontitis mouse model. FASEB J. 29, 2702-2711.

Sheridan, R. A., Wang, H. L., Eber, R. and Oh, T. J. (2015) Systemic Chemotherapeutic agents as adjunctive periodontal therapy: a narrative review and suggested clinical recommendations. J. Int. Acad. Periodontol. 17, 123-134.

Suen, P. K. and Qin, L. (2016) Sclerostin, an emerging therapeutic target for treating osteoporosis and osteoporotic fracture: a general review. J. Orthop. Translat. 4, 1-13.

Venkatramanan, M., Sankar Ganesh, P., Senthil, R., Akshay, J., Veera Ravi, A., Langeswaran, K., Vadivelu, J., Nagarajan, S., Rajendran,
K. and Shankar, E. M. (2020) Inhibition of quorum sensing and biofilm formation in Chromobacterium violaceum by fruit extracts of Passiflora edulis. ACS Omega 5, 25605-25616.

Winkler, D. G., Sutherland, M. K., Geoghegan, J. C., Yu, C. P., Hayes, T., Skonier, J. E., Shpektor, D., Jonas, M., Kovacevich, B. R., Staehling-Hampton, K., Appleby, M., Brunkow, M. E. and Latham, J. A. (2003) Osteocyte control of bone formation via sclerostin, a novel BMP antagonist. EMBO J. 22, 6267-6276.

Xue, F., Shu, R. and Xie, Y. (2015) The expression of NLRP3, NLRP1 and AIM2 in the gingival tissue of periodontitis patients: RT-PCR study and immunohistochemistry. Arch. Oral Biol. 60, 948-958.

Yada, S., Kamalesh, B., Sonwane, S., Guptha, I. and Swetha, R. K. (2015) Quorum sensing inhibition, relevance to periodontics. J. Int. Oral Health 7, 67-69.

Yao, Y., Kauffmann, F., Maekawa, S., Sarment, L. V., Sugai, J. V., Schmiedeler, C. A., Doherty, E. J., Holdsworth, G., Kostenuik, P. J. and Giannobile, W. V. (2020) Sclerostin antibody stimulates periodontal regeneration in large alveolar bone defects. Sci. Rep. 10, 16217. 